\title{
Primeira descrição da caracterização fenotípica e susceptibilidade in vitro a drogas de leveduras do gênero Cryptococcus spp isoladas de pacientes HIV positivos e negativos, Estado de Mato Grosso
}

\author{
First description of phenotypic profile and in vitro drug susceptibility \\ of Cryptococcus spp yeast isolated from HIV-positive and \\ HIV-negative patients in State of Mato Grosso
}

\author{
Olivia Cometti Favalessa', Luciano Correa Ribeiro ${ }^{2}$, Tomoko Tadano ${ }^{2}$, \\ Cor Jesus Fernandes Fontes ${ }^{2}$, Flávio Basili Dias ${ }^{1}$, Bruno Pereira Albuquerque Coelho ${ }^{1}$ \\ e Rosane Christine Hahn ${ }^{1,2}$
}

\begin{abstract}
RESUMO
Foram avaliados 37 isolados de 10 pacientes HIV negativos e 26 positivos, em Mato Grosso. Exame direto, cultura e quimiotipagem de espécies foram realizados. Cetoconazol, itraconazol, voriconazol, fluconazol e anfotericina B foram avaliados. Foram identificadas 37 leveduras do gênero Cryptococcus spp sendo 26 de pacientes HIV- positivos (25 Cryptococcus neoformans e um Cryptococcus gattii) e 10 de HIV- negativos (cinco Cryptococcus neoformans e cinco Cryptococcus gattii). Considerando isolados clínicos (Cryptococcus neoformans) de HIV positivos observouse resistência ( $8 \%$ e $8,7 \%$ ) e susceptibilidade dose-dependência (20\% e 17,4\%) para fluconazol e itraconazol respectivamente. Para isolados de Cryptococcus neoformans oriundos de pacientes HIV negativos, observou-se susceptibilidade dose-dependência (40\%) ao fluconazol. Os isolados de Cryptococcus gattii provenientes de pacientes HIV- negativos mostraram-se susceptíveis a todos os antifúngicos, exceto um isolado de Cryptococcus gattii que foi susceptível dose-dependente ao fluconazol (20\%). 0 isolado proveniente do paciente HIV- positivo demonstrou resistência ao fluconazol $(\mathrm{CIM} \geq 256 \mu \mathrm{g} / \mathrm{mL})$ e itraconazol $(\mathrm{CIM}=3 \mu \mathrm{g} / \mathrm{mL})$.
\end{abstract}

Palavras-chaves:Cryptococcus spp. HIV. Drogas antifúngicas. Mato Grosso.

\begin{abstract}
Thirty-seven isolates from 10 HIV-negative and 26 HIV-positive patients in Mato Grosso were evaluated. Direct examination, culturing and chemotyping of species were performed. Ketoconazole, itraconazole, voriconazole, fluconazole and amphotericin B were evaluated. Thirty-seven yeasts of Cryptococcus spp were identified, of which 26 were from HIV-positive patients (25 Cryptococcus neoformans and one Cryptococcus gattii) and 10 from HIV-negative patients (five Cryptococcus neoformans and five Cryptococcus gattii). The Cryptococcus neoformans clinical isolates from HIV-positive patients showed resistance ( $8 \%$ and $8.7 \%$ ) and dose-dependent susceptibility (20\% and $17.4 \%)$ to fluconazole and itraconazole, respectively. Among the Cryptococcus neoformans isolates from HIV-negative patients, there was dose-dependent susceptibility (40\%) to fluconazole. Cryptococcus gattii isolates from HIV-negative patients were shown to be susceptible to all antifungal agents, except for one isolate of Cryptococcus gattii that showed dose-dependent susceptibility to fluconazole (20\%). The Cryptococcus gattii isolate from an HIV-positive patient showed resistance to fluconazole (MIC $\geq 256 \mu \mathrm{g} / \mathrm{ml}$ ) and itraconazole (MIC $=3 \mu \mathrm{g} / \mathrm{ml}$ ).
\end{abstract}

Key-words: Cryptococcus spp. HIV. Antifungal drugs. Mato Grosso.

1. Laboratório de Investigação (Micologia), Faculdade de Ciências Médicas, Universidade Federal de Mato Grosso, Cuiabá, MT. 2. Hospital Universitário Júlio Müller, Universidade Federal de Mato Grosso, Cuiabá, MT.

Endereço para correspondência: Prof ${ }^{ \pm}$Rosane Hahn. Faculdade de Ciências Médicas/UFMT. Av. Fernando Corrêa da Costa 2367, Boa Esperança 78060-900 Cuiabá, MT.

Tel: 5565 3615-8809

e-mail: rchahn@terra.com.br

Recebido para publicação em 30/03/2009

Aceito em 19/10/2009
A criptococose vem assumindo um papel relevante na história da AIDS, e vem sendo considerada uma das micoses oportunistas mais comuns diagnosticadas entre os pacientes infectados pelo HIV. É uma doença de caráter sistêmico e tem sido uma das principais responsáveis pela morbidade e mortalidade neste grupo de pacientes. A meningite é a apresentação clínica mais frequiente $e^{1127}$.

No período de 1980 a 2002, 6\% das infecções oportunistas, presentes em pacientes portadores do HIV, foram ocasionadas 
pelo Cryptococcus neoformans ${ }^{22}$, sendo a criptococose a segunda infecção oportunista mais comum diagnosticada nestes pacientes $^{23}$.

A criptococose é causada principalmente por duas espécies do complexo Cryptococcus neoformans, Cryptococcus neoformans (sorotipo A, D e AD) e Cryptococcus gattii (sorotipo B e C) ${ }^{19}$. 0 Cryptococcus neoformans apresenta distribuição cosmopolita sendo comumente encontrado na natureza e, essa espécie é frequentemente associada a fezes de pombos urbanos e outras aves, assim como vegetais, madeira e no solo ${ }^{17233228}$. Em indivíduos imunocomprometidos, a espécie mais prevalente é Cryptococcus neoformans ${ }^{6141517}$. Já o Cryptococcus gattii apresenta distribuição limitada, sendo encontrado no meio ambiente, principalmente em regiões tropicais e semitropicais, sendo o primeiro isolamento descrito em 1990 em Eucalyptus camadulensis ${ }^{12}{ }^{13} 32$. Em geral, acomete indivíduos imunocompetentes ${ }^{615} 19$.

Com o surgimento de cepas resistentes e reincidência das infecções fúngicas invasivas quando submetidas à terapêutica clássica, atualmente empregada, tem sido reconhecida a importância dos testes de susceptibilidade in vitro para auxiliar/redirecionar o tratamento e acompanhar a evolução da terapia antifúngica para cada caso ${ }^{59}$. Existem vários fatores que podem contribuir para ocorrência de falha terapêutica, assim como podem existir fenótipos resistentes à terapêutica empregada. Os fenótipos resistentes podem ser identificados em uma população contendo vários agentes etiológicos por meio de testes de susceptibilidade in vitro os quais podem ser realizados por vários métodos disponíveis no mercado ${ }^{25}$.

Os testes de susceptibilidade in vitro vêm sendo realizados com maior frequência, e várias técnicas tem sido desenvolvidas e empregadas para o estudo do perfil de susceptibilidade in vitro aos antifúngicos. Dentre as opções existentes, o método de microdiluição em caldo é considerado o método de referência, documento M27-A2 (2005) do Clinical Laboratory Standards Institute (CLSI). Métodos alternativos estão sendo implantados para facilitar a utilização e implantação destes testes na rotina laboratorial, destacando-se o Etest. Este método compreende uma técnica de fácil e rápida execução e, apresenta satisfatória correlação com o método de referência (CLSI) ${ }^{4}$. As drogas antifúngicas mais utilizadas no tratamento das manifestações graves da criptococose em pacientes imunodeprimidos são: anfotericina B, 5-fluocitosina e fluconazol ${ }^{30}$.

Objetivamos neste estudo avaliar a importância da caracterização das leveduras do gênero Cryptococcus em pacientes HIV positivos e negativos, assim como determinar o perfil de susceptibilidade in vitro aos antifúngicos. Vale ressaltar que no Estado do Mato Grosso (Centro-Oeste do Brasil), este estudo apresenta caráter inédito.

\section{MATERIAL E MÉTODOS}

Amostras biológicas de pacientes HIV positivos e negativos com criptococose. Estudo tipo descritivo prospectivo realizado no período compreendido entre janeiro de 2005 a outubro de 2008. Foram estudadas 37 amostras clínicas diferentes: líquido cefalorraquidiano (LCR), sangue venoso periférico, lavado broncoalveolar (LBA), aspirado de linfonodo de membro superior direito (MSD), todas provenientes de pacientes HIV positivos e negativos com diagnóstico clínico e laboratorial de criptococose. 0 presente estudo foi aprovado pelo comitê de ética em pesquisa (CEP) envolvendo seres humanos do Hospital Universitário Julio Müller da Universidade Federal de Mato Grosso (HUJM/UFMT), sob o número 247/06. Foram incluídas neste estudo quatro unidades hospitalares, correspondendo a dois hospitais universitários, pronto socorro municipal de Cuiabá, e pronto socorro municipal de Várzea Grande.

Dados clínicos. Os pacientes HIV positivos incluídos no estudo tinham diagnóstico confirmado de infecção pelo HIV, e encontravam-se em estágio avançado da doença. Os pacientes soronegativos também foram confirmados quanto à ausência do HIV. Todos os pacientes avaliados neste estudo assinaram o termo de consentimento livre e esclarecido aprovado pelo CEP/HUJM.

Identificação das espécies de leveduras do gênero Cryptococcus. Inicialmente, foi realizado o exame direto com tinta nanquim, e em seguida foi realizado o isolamento do fungo em ágar Sabouraud dextrose (Difco-USA). Os isolados apresentando colônias de consistência cremosa sugestivas de Cryptococcus spp foram repicadas em meio ágar niger para confirmação deste gênero. As espécies classificadas através das etapas anteriores como pertencentes ao gênero Cryptococcus foram caracterizadas em meioL-canavanina-glicina-azul de bromotimol (CGB) ${ }^{18}$ como Cryptococcus neoformans e Cryptococcus gattii.

Teste de susceptibilidade aos antifúngicos. 0 perfil de susceptibilidade foi realizado pelo Etest (AB Biodisk) seguindo as instruções do fabricante ${ }^{1}$. Como não são referenciados pontos de corte para Cryptococcus neoformans e Cryptococcus gattii pelo CLSI, foram utilizados aqueles empregados por outros autores ${ }^{262931}$. Os antifúngicos testados foram cetoconazol, fluconazol, itraconazol, voriconazol e anfotericina $\mathrm{B}$ (AB Biodisk).

Controle de qualidade. A atividade das drogas antifúngicas e a reprodutibilidade dos resultados foram asseguradas pela inclusão dos isolados de referência Candida parapsilosis - ATCC 22019 e Candida krusei - ATCC 6258, que foram gentilmente cedidas pela Dra. Márcia S. C. Melhem do Instituto Adolfo Lutz, SP.

\section{RESULTADOS}

Considerando os pacientes HIV positivos, dos 26 isolados de leveduras do gênero Cryptococcus, 15 pertenciam ao sexo masculino e 9 ao sexo feminino. Foram caracterizados 25 (96,1\%) Cryptococcus neoformans e um (3,8\%) Cryptococcus gattii , com média de idade igual a 40,2 anos $(\mathrm{DP}=8,8)$.

Considerando os pacientes HIV-negativos, dos 10 isolados de leveduras do gênero Cryptococcus, quatro pertenciam ao sexo masculino e seis ao sexo feminino. Foram caracterizados cinco (50\%) Cryptococcus neoformans e cinco (50\%) Cryptococcus gattii, e a média de idade foi de 31 anos $(\mathrm{DP}=13,9)$.

Nas Tabelas 1 e 2, estão apresentados os valores das concentrações inibitória mínimas (CIM) para inibição de $50 \%\left(\mathrm{CIM}_{50}\right)$ e $90 \%\left(\mathrm{CIM}_{90}\right)$ e suas variações referentes aos 
TABELA 1

Concentração inibitória mínima das cinco drogas antifúngicas frente às espécies de leveduras do gênero Cryptococcus de pacientes HIV- positivos.

\begin{tabular}{|c|c|c|c|c|c|c|c|}
\hline \multirow[b]{2}{*}{ Cryptococcus spp } & \multirow[b]{2}{*}{ Antifúngico } & \multirow[b]{2}{*}{ Variação } & \multicolumn{2}{|c|}{ CIM $(\mu \mathrm{g} / \mathrm{ml})$} & \multicolumn{3}{|c|}{ Perfil de susceptibilidade } \\
\hline & & & $50 \%$ & $90 \%$ & $\mathrm{~S}(\%)$ & SDD (\%) & $\mathrm{R}(\%)$ \\
\hline \multirow{5}{*}{ Cryptococcus neoformans } & cetoconazol & 0,064 a 1,5 & 0,5 & 0,75 & 100,0 & - & 0,0 \\
\hline & fluconazol & $1,00 a>256$ & 816 & 72,0 & 20,0 & 8,0 & \\
\hline & itraconazol & 0,008 a 1,5 & 0,094 & 0,38 & 73,9 & 16,0 & 8,0 \\
\hline & voriconazol & 0,012 a 0,25 & 0,032 & 0,064 & 100,0 & 0,0 & 0,0 \\
\hline & anfotericina $\mathrm{B}$ & 0,094 a 1,0 & 0,50 & 1,00 & 100,0 & 0,0 & 0,0 \\
\hline
\end{tabular}

S: susceptível, SDD: susceptível dose-dependente, R: resistente.

$\mathrm{CIM}_{30}$ e $\mathrm{CIM}_{\text {gp }}$ concentração capaz de inibir o crescimento dos isolados em $50 \%$ e $90 \%$, respectivamente.

TABELA 2

Concentração inibitória mínima das cinco drogas antifúngicas frente às espécies de leveduras do gênero Cryptococcus isolados de pacientes HIV- negativos.

\begin{tabular}{|c|c|c|c|c|c|c|c|}
\hline \multirow[b]{2}{*}{ Antifúngico } & \multirow[b]{2}{*}{ Cryptococcus spp } & \multirow[b]{2}{*}{ Variação } & \multicolumn{2}{|c|}{ CIM $(\mu \mathrm{g} / \mathrm{ml})$} & \multicolumn{3}{|c|}{ Perfil de susceptibilidade } \\
\hline & & & $50 \%$ & $90 \%$ & S (\%) & SDD (\%) & $\mathrm{R}(\%)$ \\
\hline \multirow[t]{2}{*}{ Cetoconazol } & Cryptococcus neoformans & $0,094-0,50$ & 0,38 & 0,5 & 100,0 & - & - \\
\hline & Cryptococcus gattii & $0,125-0,50$ & 0,19 & 0,38 & 100,0 & - & - \\
\hline \multirow[t]{2}{*}{ Fluconazol } & Cryptococcus neoformans & $3,0-32,0$ & 4 & 16 & 60,0 & 40,0 & - \\
\hline & Cryptococcus gattii & $3,0-16,0$ & 6 & 12 & 80,0 & 20,0 & - \\
\hline \multirow[t]{2}{*}{ Itraconazol } & Cryptococcus neoformans & $0,032-0,094$ & 0,032 & 0,047 & 100,0 & - & - \\
\hline & Cryptococcus gattii & $0,016-0,094$ & 0,023 & 0,047 & 100,0 & - & - \\
\hline \multirow[t]{2}{*}{ Voriconazol } & Cryptococcus neoformans & $0,016-0,094$ & 0,032 & 0,047 & 100,0 & - & - \\
\hline & Cryptococcus gattii & $0,016-0,064$ & 0,023 & 0,032 & 100,0 & - & - \\
\hline \multirow[t]{2}{*}{ Anfotericina B } & Cryptococcus neoformans & $0,38-1,5$ & 0,38 & 1 & 100,0 & - & - \\
\hline & Cryptococcus gattii & $0,094-0,50$ & 0,25 & 0,50 & 100,0 & & \\
\hline
\end{tabular}

S: susceptível, SDD: susceptibilidade dose-dependente, R: resistente.

$\mathrm{CIM}_{90}$ e $\mathrm{CIM}_{90}$ concentração capaz de inibir o crescimento dos isolados em $50 \%$ e $90 \%$, respectivamente.

antifúngicos testados frente aos isolados Cryptococcus spp dos pacientes HIV positivos e negativos. Considerando os pacientes HIV-positivos, a maioria dos isolados de Cryptococcus neoformans mostrou-se susceptível aos cinco antifúngicos testados. Porém, $8 \%$ mostraram-se resistentes e $20 \%$ susceptíveis dose-dependentes frente ao fluconazol. Para o itraconazol, $8 \%$ mostraram-se resistentes e 16\% susceptíveis dose-dependentes. Os valores de CIM $(\mu \mathrm{g} / \mathrm{mL})$ para o isolado de Cryptococcus gattii foram: cetoconazol $=3,0$; fluconazol $>256$; itraconazol $=$ 3,0; voriconazol $=0,50$ e anfotericina $B=0,064$, demonstrando resistência ao fluconazol e itraconazol.

Os isolados de Cryptococcus neoformans provenientes de pacientes HIV-negativos mostraram susceptibilidade in vitro aos antifúngicos, sendo que $40 \%$ destes isolados foram susceptíveis dose-dependentes ao fluconazol e $20 \%$ resistentes à anfotericina B. Considerando a espécie, Cryptococcus gattii, todos os isolados foram susceptíveis a todos os antifúngicos, exceto um, o qual apresentou $20 \%$ de susceptibilidade dose-dependência frente ao fluconazol.

Quanto ao local de isolamento, considerando pacientes soropositivos, os isolados de Cryptococcus neoformans de LCR apresentaram valores de CIM mais elevados para todos os antifúngicos testados quando comparados aos outros sítios de isolamento, exceto para anfotericina B (Tabela 3).
TABELA 3

Variação da concentração inibitória mínima quanto ao local de isolamento das amostras de Cryptococcus neoformans isolados de pacientes HIV- positivos.

Variação da CIM $(\mu \mathrm{g} / \mathrm{ml})$

Local de isolamento do Cryptococcus neoformans (n⿳o de amostras)

\begin{tabular}{|c|c|c|c|c|}
\hline Antifúngicos & $\begin{array}{c}\text { LCR } \\
n^{0}=17\end{array}$ & $\begin{array}{c}\text { Sangue } \\
\mathrm{n}^{0}=6\end{array}$ & $\begin{array}{c}\text { Escarro } \\
\mathrm{n}^{0}=1\end{array}$ & $\begin{array}{l}\text { LMSD } \\
\mathrm{n}^{0}=1\end{array}$ \\
\hline Cetoconazol & $0,064-1,5$ & $0,25-0,75$ & 0,5 & 0,25 \\
\hline Fluconazol & $1,0->256$ & $2,0-16,0$ & 12 & 2 \\
\hline Itraconazol & $0,008-1,5$ & $0,064-0,25$ & 0,19 & 0,012 \\
\hline Voriconazol & $0,012-0,25$ & $0,016-0,064$ & 0,064 & 0,023 \\
\hline Anfotericina B & $0,094-1,0$ & $0,38-1,0$ & 0,38 & 0,75 \\
\hline
\end{tabular}

LMSD: aspirado de linfonodo de membro superior direito.

LCR: líquido cefalorraquidiano.

\section{DISCUSSÃO}

Neste trabalho, os isolados clínicos de Cryptococcus neoformans em sua maioria apresentaram-se susceptíveis aos cinco antifúngicos testados; porém, detectamos isolados resistentes ao fluconazol e itraconazol, considerando pacientes HIV-positivos, como ilustra a Tabela 1. Os isolados de pacientes HIV-negativos também se mostraram susceptíveis em sua maioria, e apenas um isolado de Cryptococcus neoformans e 
um de Cryptococcus gattii apresentaram susceptibilidade dosedependência ao fluconazol.

Anfotericina B tem sido utilizada para o tratamento de diversas infecções por fungos micelianos e leveduriformes, em que pese a sua nefrotoxicidade ${ }^{8}$. Como ainda não foram definidos os pontos de corte para a anfotericina B, segundo o CLSI, foi utilizado neste estudo aqueles empregados por Nguyen e $\mathrm{Yu}^{24}$ e Lozano cols ${ }^{20}$. Eles definiram resistência à anfotericina $\mathrm{B}$ com valores de CIM iguais ou superiores a $2 \mu \mathrm{g} / \mathrm{mL}$.

Em relação aos derivados azólicos, tem sido observado que a profilaxia utilizada com fluconazol para pacientes infectados pelo vírus HIV, pode ser apontada como um dos fatores responsáveis pela elevação de concentrações inibitórias mínimas in vitro. No entanto, a boa penetração desta droga no liquido cefalorraquidiano, justifica os bons resultados observados in vivo ${ }^{16}$.

Os valores encontrados neste estudo apontam melhor atividade do voriconazol frente ao itraconazol e fluconazol para os isolados de Cryptococcus neoformans em pacientes HIV positivos. Considerando os HIV-negativos foi possível observar semelhança quanto ao perfil de susceptibilidade entre itraconazol e voriconazol. Apesar dos resultados promissores in vitro para o voriconazol, há necessidade da busca de correlação com a prática clínica ${ }^{16}$. Ainda em 1998, Nguyen e $\mathrm{Yu}^{24}$ demonstraram a superioridade da atividade in vitro do voriconazol frente ao itraconazol e fluconazol, corroborando os dados encontrados neste trabalho.

A resistência observada in vitro para isolado de Cryptococcus gattii, neste trabalho, pode ser comparada aquela observada em 1999 por De Bedout cols ${ }^{10}$, os quais observaram que para 15 isolados de Cryptococcus gattii oriundos da Colômbia, foram observadas concentrações inibitórias mínimas mais elevadas para anfotericina B, itraconazol e fluconazol. Este resultados indicam que, in vitro, estes antifúngicos não exercem uma ação inibitória tão marcante como era de se esperar. Este fato pode apontar a necessidade de terapia mais prolongada para os pacientes infectados por esta espécie.

A correlação clínico-laboratorial foi relatada por Aller cols ${ }^{2}$, considerando valores de concentrações inibitórias mínimas de fluconazol e fracasso terapêutico em infecções criptocócicas. Estes autores avaliaram 28 isolados clínicos de Cryptococcus neoformans provenientes de 25 pacientes com AIDS. Os resultados obtidos mostraram que os valores de $\mathrm{CIM}_{50}$ e $\mathrm{CIM}_{90}$ corresponderam a 4 e $16 \mu \mathrm{g} / \mathrm{mL}$ respectivamente. Dos 25 pacientes estudados, 4 morreram com infecção criptocócica ativa e 2 por outra causas. Insucesso terapêutico foi observado em 5 pacientes os quais foram infectados por isolados que exibiram valores de

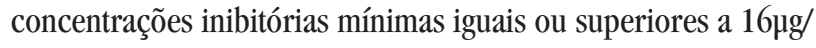
$\mathrm{mL}$. Estes dados sugerem valores de concentrações inibitórias mínimas como preditoras de resposta terapêutica.

Em 2001, um grupo de pesquisadores brasileiros registrou a menor atividade in vitro dos isolados clínicos de Cryptococcus neoformans frente ao fluconazol em relação aos isolados ambientais $^{3}$.
Em relação ao método utilizado (Etest) neste estudo para determinação da atividade in vitro de drogas antifúngicas, vale ressaltar que Mawwell cols ${ }^{21}$ determinaram o perfil de susceptibilidade de 162 isolados clínicos de Cryptococcus neoformans. Estes autores sugeriram que o Etest constitui um método útil para determinação do perfil de susceptibilidade especialmente para o voriconazol e anfotericina $\mathrm{B}$. Chandenier cols ${ }^{6}$ também confirmam o valor do Etest para avaliar in vitro isolados de Cryptococcus neoformans frente ao voriconazol.

Considerando dados da região Centro-Oeste, registrados por Fernandes cols ${ }^{14}$ foi possível verificar resistência ao fluconazol tanto para os isolados de Cryptococcus neoformans como de Cryptococcus gattii, como observado neste estudo. Estes dados evidenciam a importância não só quanto à determinação da espécie do gênero Cryptococcus, como também do perfil in vitro de susceptibilidade.

Apesar da detecção neste trabalho de apenas um isolado de Cryptococcus gattii resistente ao fluconazol e itraconazol, Trilles cols $^{33}$ mostraram que mais de $90 \%$ dos isolados desta mesma espécie exibiram concentrações inibitórias mínimas maiores que $16 \mu \mathrm{g} / \mathrm{mL}$ e $64 \mu \mathrm{g} / \mathrm{mL}$ ao itraconazol e fluconazol respectivamente para 57 isolados avaliados. Os resultados obtidos por estes autores apontam menor susceptibilidade de Cryptococcus gattii frente aos antifúngicos testados quando comparados a Cryptococcus neoformans, conforme outros registros encontrados ${ }^{33}$.

Em síntese, os resultados obtidos neste estudo apontam a maior prevalência de Cryptococcus neoformans como causa de criptococose, e maior número de isolados desta espécie susceptíveis ou susceptíveis dose-dependentes. Entretanto, monitorar o perfil de susceptibilidade in vitro às drogas antifúngicas em algumas situações clínicas merece olhar atento por parte dos infectologistas, em casos de insucesso à terapêutica clássica utilizada.

\section{REFERÊNCIAS}

1. AB Biodisk. Etest Technical guide 4b: Antifungal susceptibility testing of yeasts. Piscataway: AB Biodisk, New Jersey, 1994.

2. Aller AI, Martin-Mzuelos E, Lozano F, Gomez_Mateos J, Steele-Moore L, Holloway WJ, Gutiérrez MJ, recio FJ, Espinel-Ingrof A. Correlation of Fluconazol MICs with Clinical Outcome in Cryptococcal infection. Antimicrobial Agents and Chemotherapy 44: 1544-1548, 2000.

3. Alves SH, Oliveira LT, Costa JM, Lubeck I, Casali AK, Vainstein MH. In vitro susceptibility to Antifungal Agents of Clinical and Environmental Cryptococcus neoformans isolated in Southern of Brazil. Revista do Instituto de Medicina Tropical de São Paulo 43: 267-270, 2001.

4. Ballesté R, Arteta Z, Barloco A, Mier C, Fernández N, Mousqués N, Xavier B, Cabrera MJ, Combol A, Gezuele E. Evaluación Del desempeño diagnóstico Del médio de difusión em Agar Etest para El estúdio de sensibilidad a los antifúngicos. Revista Medica Del Uruguay 22:128-135, 2006.

5. Calhoun DL, Roberts GD, Galgiani JN, Bennett JE, Feingold DS, Jorgensen J, Kobayashi GS, Shadomy S. Results of survey of antifungal susceptibility tests in the United States and interlaboratory comparison of broth dilution testing of flucytosine and amphotericin. Bangladesh Journal Clinical Microbiology 23:298301, 1986.

6. Chandenier J, Adou-Bryan KD, Douchet C, Sar B, Kombila M, Swinne D, ThérizolFerly M, Buisson Y, Richard-Lenoble D. In vitro activity of amphotericin B, Fluconazol and Voriconazole against 162 Cryptococcus neoformans isolates 
from Africa and Cambodia. European Journal Clinical Microbiology Infection Diseases 23: 506-508, 2004.

7. Chen S, Sorrell T, Nimmo G, Speed B, Currie B, Ellis D, Marriott D, Pfeiffer T, Parr D, Byth K. Australasian Cryptococcal Study Group Epidemiology and Host- and variety-dependent characteristics of infection due to Cryptococcus neoformans in Australia and New Zealand. Clinical Infectious Diseases 31: 499-508, 2000.

8. Chen SC, Sorrell TC. Antifungal agents: new drugs, old drugs. Medical Journal of Australia 187: 404-409, 2007.

9. Colombo AL, Barchiesi F, Mcgough DA, Rinald MG. Comparison of Etest and National Committee for Clinical Laboratory Standards broth macrodilution method for azole antifungal susceptibility testing. Journal of Clinical Microbiology 33:535-540, 1995.

10. De Bedout C, Ordónez N, Gómez BL, Rodriguez MC, Arango M, Restrepo A, Castaneda. In vitro antifungal susceptibility of clinical isolates of Cryptococcus neoformans var. neoformans and Cryptococcus neoformans var. gattii. Revista Iberoamericana de Micologia 16:36-39, 1999

11. Dismukes WE. Cryptococcal meningitis in patients with AIDS. Journal Infection Diseases 157:624.628, 1988

12. Ellis DH, Pfeiffer TJ. Natural Habitat of Cryptococcus neoformans var. gattii. Journal of Clinical Microbiology 25:430-431, 1990.

13. Ellis DH, Pfeiffer TJ. Ecology, life cycle, and infectious propagule of Cryptococcus neoformans. Lancet 336: 923-925, 1990.

14. Fernandes OFL, Costa TR, Costa MR, Soares AJ, Pereira AJSC, Silva MR. Cryptococcus neoformans isolados de pacientes com AIDS. Revista da Sociedade Brasileira de Medicina Tropical 33: 75-78, 2000.

15. Fromtling RA, Bulmer GS. Distribution of Cryptococcus neoformans in a natural site. Infection and Immunity 31: 560-563, 1981

16. Illnait-Zaragozi MT, Martínez GF, Curfs-Breuker I, Fernadez CM, Boekhout T, Meids JF. In vitro activity of the New Azole Isavuconazole (BAL4815) compared with six other Antifungal Agents against 162 Cryptococcus neoformans isolates from Cuba. Antimicrobial Agents and Chemotherrapy 52: 1580-1582, 2008.

17. Kobayashi CCBA, Souza LKH, Fernandes OFL, Brito SCA, Silva AC, Souza ED, Silva MRR. Characterization of Cryptococcus neoformans isolated from urban environmental sources in Goiânia, Goiás State, Brazil. Revista do Instituto Medicina Tropical São Paulo 47: 203-207, 2005.

18. Kwon-Chung KJ, Polacheck I, Bennett JE. Improved diagnostic Medium for Separation of Cryptococcus neoformans var. neoformans (Serotypes A and D) and Cryptococcus neoformans var. gattii (Serotypes B and C). Journal of Clinical Microbiology 15:535-537, 1982.

19. Lindenberg ASC, Chang MR, Paniago AMM, Lazéra MS, Moncada PMF, Bonfim GS, Nogueira AS,Wanke B. Clinical and Epidemiological features of 123 cases of cryptococcosis in Mato Grosso do Sul, Brazil. Revista do Instituto de Medicina Tropical 50: 75-78, 2008.

20. Lozano-Chiu MVL, Paetznick MA, Gannoum Rex JH. Detection of resistance to amphotericin B among Cryptococcus neoformans clinical isolates: performances of three different media assessed by using E-test and National Committee for Clinical Laboratory Standards M27-A methodology. Journal of Clinical Microbiology 36: 2817-2822, 1998

21. Maxwell MJ, Messer SA, Hollis RJ, Dieckma DJ, Pfaller MA. Evaluation of Etes Method for Determining Voriconazole and Amphotericin B MICs for 162 Clinical Isolates of Cryptococcus neoformans. Journal of Clinical Microbiology 41: 97-99, 2003

22. Ministério da Saúde. Dados e Pesquisa em DST e Aids. Coordenação do programa nacional de DST/Aids. Boletim Epidemiológico. AIDS/Ministério da Saúde. Brasília, 2002.

23. Mitchell TG, Perfect JR. Cryptococcosis in the era of AIDS - 100 years after the discovery of Cryptococcus neoformans. Clinical Microbiology and Infection 8: 515-548, 1995.

24. Nguyen $\mathrm{MH}, \mathrm{Yu} \mathrm{CY}$. In vitro comparative efficacy of vorconazole nd itrconzole against fluconazole-susceptible and resistant Cryptococcus neoformans isolates. Antimicrobial Agents Chemotherapy 42: 471-472, 1998.

25. Pappalardo MCSM, Melhem MSC. Cryptococcosis: A Review of the Brazilian Experience for the Disease. Revista do Instituto de Medicina Tropical 45:299-305, 2003.

26. Pedroso RS, Ferreira JC, Candido RC. In vitro susceptibility antifungal agents of environmental Cryptococcus spp, isoled in the city of Ribeirão Preto, São Paulo, Brazil. Memórias do Instituto Osvaldo Cruz 101: 239-243, 2006.

27. Powderly WG. Cryptococcal meningitis and AIDS. Clinical Infection Diseases 17:837-842, 1993

28. Reolon A, Perez LRR, Mezzari A. Prevalência de Cryptococcus neoformans nos pombos urbanos da cidade de Porto Alegre, Rio Grande do Sul. Jornal Brasileiro de Patologia e Medicina Laboratorial 40: 293-298, 2004.

29. Rodriguez-Tudela JL, Berenguer Martinez-Suarez JJ, Sanchez R. Comparision of a Spectrophotometric Microdilution Method with RPMI-2\% Glucose with the National Committee for Clinical Laboratory Standards Reference Macrodiluition Method M27-P for in vitro Susceptibility Testing of Amphotericin B, Flucytosine, and Fluconazol against Candida albicans. Antimicrobial and Chemotherapy 40: 1998-2003, 1996.

30. Saag MS, Graybill RJ, Larsen RA, Pappas PG, Perfect JR. Powderly WG, Sobel JD, Dismukes WE. Practice guidelines for the management of cryptococcal disease. Infectious Diseases Society of American. Clinical Infection Diseases 30: 710-718, 2000.

31. Souza LKH, Fernandes OFL, Kobavashi CCBA, Passos XS, Costa CR, Lemos JA, SouzaJúnior AH, Silva MRR. Antifungal susceptibilities of clinical and environmental isolates of Cryptococcus neoformnas in Goiânia City, Goiás, Brazil. Revista do Instituo de Medicina Tropical de São Paulo 47: 253-256, 2005.

32. Sorrel TC, Ellis DH. Ecology of Cryptococcus neoformans. Revista Iberoamerican de Micologia 14:42-43, 1997

33. Trilles L, Fernández-Torres B, Lazéra MS, Wanke B, Guarro J. In vitro Antifungal Susceptibility of Cryptococcus gattii. Journal of Clinical Microbiology 42: 4815-4817, 2004 